\title{
The Qualitative Comparative Analysis: An Overview of a Causal Complexity Approach
}

\author{
Monika Smela ${ }^{1, *}$ \\ ${ }^{1}$ University of Economics, Faculty of International Relations, Department of World Economics, \\ nam.W. Churchilla 1938/4, 13067 Prague 3, Czech Republic
}

\begin{abstract}
.
Research background: Alongside with the development of configurative comparative analysis aiming at identification of necessary and sufficient conditions, various formal methods used for this purpose have been formulated during the last decades. One of them is qualitative comparative analysis (QCA), one of approaches used for causal explanation of phenomena of cases performed in the field of international economics and global affairs.

Purpose of the article: The main purpose of the article is to provide a detailed overview of the QCA method in global context, to define its methodologic foundations and consequently introduce the key concepts of the method. The article also provides a comparison of QCA to typical tools of qualitative and quantitative approaches. On the basis of this part, both pros and cons of QCA are derived.

Methods: Basically, the methods of analysis, deduction and comparison are used to fulfil the purpose of the article. The existing and available papers and books coping with the topic of QCA and its position among other research methods are reviewed to provide an overview on the selected method.

Findings \& Value added: The QCA is a method based on analysing stated relations. It bridges the quantitative and qualitative research and reveals certain patterns based on causal complexity principles, however, it is done regarding heterogeneity and diversity of individual researched cases. It is a method applicable to the middle number of cases, it means too few cases for statistical methods on the other hand too many cases for typical qualitative approaches.
\end{abstract}

Keywords: comparative analysis; QCA method; causal complexity; necessary and sufficient conditions

JEL Classification: $B 40 ; B 41 ; C 20$

*Corresponding author: $\underline{x s m e m 28 @ v s e . c z}$ 


\section{Introduction}

Alongside with the innovative ideas on qualitative methodology in late 1960s, configurative comparative approach came into existence and lately the method of qualitative comparative analysis (QCA) was introduced by Charles Ragin in 1980s. Formally it was developed as a research method mainly for purposes of comparative politics and social sciences [1]. However, during the time, especially in the last decade, the method was formalized into an analytic approach which is nowadays commonly used by many researchers and scientists from various fields and academic areas, e. g. business research, sociology as well as economics [2].

As a configurative method, the QCA uses formalization procedures as well as it relies on Boolean logic, for this reason, it is not a typical qualitative nor quantitative method [3]. It is ranked among so called theory-based approaches whose main aim is to evaluate varied combinations of explanatory factors (conditions) explaining presence, or on the contrary absence, of a certain phenomenon (outcome). That is why, it is extremely popular in disciplines dealing with quantitative data providing in-depth information.

Due to increasing global interest in this case-based method, an overview of its basic foundations and concepts is performed. Being familiar with the main principles of each research method, including the QCA, is a key step to perform analysis in an appropriate way. The methodologic foundations of QCA are summed up in the following chapter of the paper. Moreover, it is crucial to embed the qualitative comparative analysis into generally known sorting of qualitative and quantitative research methods to understand its position among these methods. The third chapter deals with this issue and it also derives pros and cons of using QCA.

\section{Methodologic foundations of QCA}

To use this method, three basic conditions need to be fulfilled. Firstly, there must be an indication of possibility that the researched phenomenon can be evaluated in the most appropriate way by complex causal context and using the QCA procedure. Secondly, it is necessary to coordinate theory and methodology, so if the QCA comes from the theory of sets, also the research theory should come up from sets [4]. The last condition is associated with the sample, which should be of middle size comprising from 10 to 50 individual cases.

Contrasting the statistical methods, the individual cases are understood in a holistic way, for this reason the emphasis is put on individual causal conditions forming together the whole system. This fact corresponds to principles of causal complexity, allowing different conditions represented by independent variables lead to the same outcomes, referring dependent variables [5]. Theory of causal complexity contrasts causal homogeneity, a situation when a dependent variable is explained entirely by an independent variable.

The QCA method can cover the causal complexity principles because of three mutually connected concepts as follows: causal asymmetry, conjunctural causation and lastly equifinality [6]. Causal symmetry highlights the fact that despite a combination of certain conditions leads to a certain outcome, the absence of the same combination will not automatically result in outcome absence. For example, diminishing trade barriers can result in higher globalization level, however, keeping some trade barriers does not guarantee lower globalization level.

Conjunctural causation is based on the fact, that each condition influences the outcome only if it is combined with one or more different conditions. To simplify this statement, it is more probable, that a combination of conditions leads to a certain outcome. Taking into consideration the previously mentioned example, the higher level of globalization is 
produced by combination of conditions, including diminishing trade barriers, increasing foreign direct investments, taking part in developing aid and many others. The concept of conjunctural causation is supplemented by equifinality outlining that various conditions, alternatively their combinations, can result in identical final outcome. Basically, many ways how to reach higher globalization levels can be found with or without existence of trade barriers.

As it was already mentioned before, the QCA method does not aim at establishing particular effects of a chosen variable. This method points at finding logical connection among causal conditions, or their combinations. This process is based on relations and logic of sufficiency and necessity. Therefore, researchers desire to answer via QCA questions aiming at finding out what conditions, or their combinations, are necessary or sufficient to generate an outcome.

In the scope of qualitative comparative studies, the sufficient and necessary conditions are defined using the three above mentioned concepts. A causation is defined as a necessary one, if it must be present for an outcome to occur. On the contrary, sufficient causation arrives at the moment when it can produce a certain outcome itself. On the basis of this, the necessary condition is defined as a situation when a certain outcome is present, consequently a necessary condition is present as well [7]. However, at the moment when a certain outcome does not occur, the necessary outcome can or cannot be present which is derived from the application of causal asymmetry.

If a sufficient condition is present, a certain outcome is present. this is the way, how the concept of sufficient condition is defined. In the case of its absence, the outcome can or cannot be present which is grounded in the assumption of equifinality. All the mentioned relations in this part are graphically illustrated for better understanding in Fig. 1.
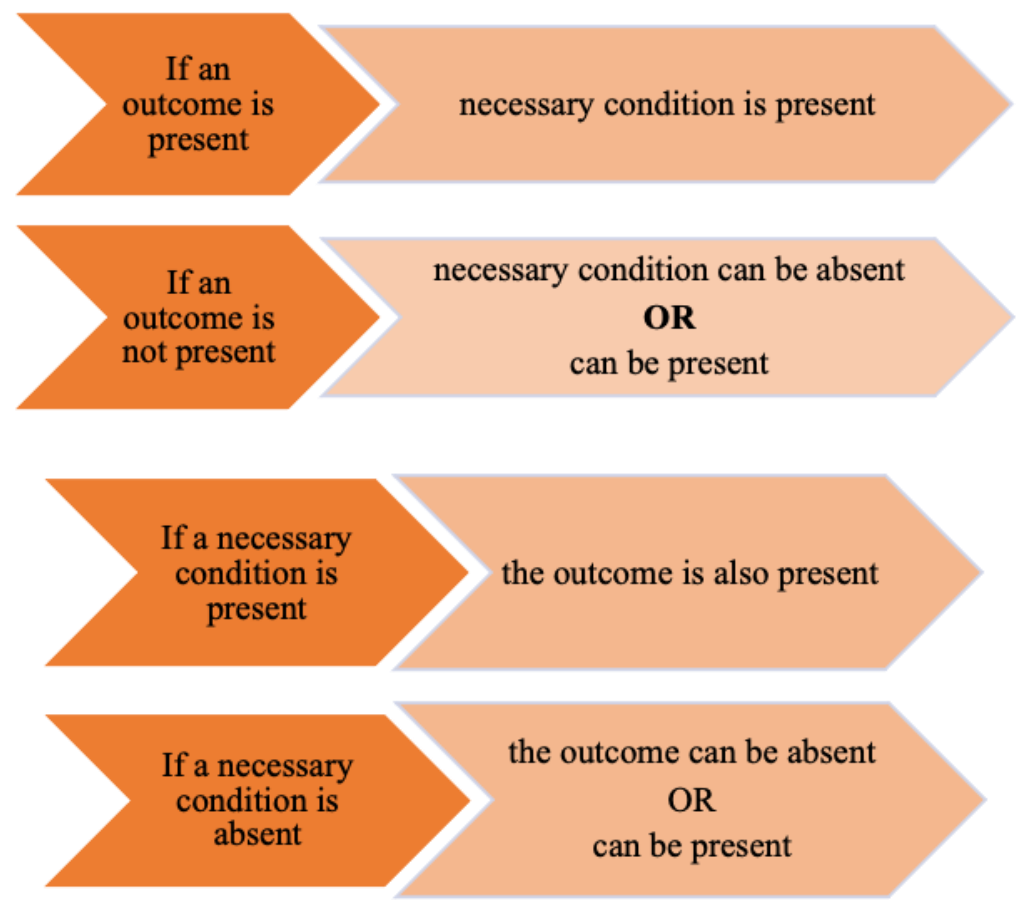

Fig. 1. Illustration of necessary and sufficient conditions in causal complexity principle 


\section{QCA within qualitative and quantitative research methods}

Charles Ragin, the founder of the QCA, and his followers managed to formulate an approach addressing limitations of both qualitative and quantitative research methods, while keeping their strong features. Thanks to it, the QCA offers a unique possibility to analyse complex relations between conditions and an outcome.

The previous chapter shows that the QCA has its foundations in set-theory and formal logic, contrasting to qualitative research based on induction and deduction or quantitative research typically grounded in regression and correlation. Qualitatively orientated methods are also case-oriented, like the QCA, however, they deal with a single case or a few ones [8]. On the contrary, the quantitative researches cope with so called large-N, meaning large sample sizes, but they are rather variable orientated. So QCA approach deals with social phenomena that used to be unresearched because of methodological gap in the past. Nowadays, QCA offers a possibility to explore also these issues.

One value added of QCA is the fact that it enables to conduct a cross-case comparison systematically and to find out complex causality in medium-N case studies. The qualitative methods using only a few cases cannot generate such a possibility of comparison among individual cases. The QCA preserves cases as holistic units through the analysis, thanks to which it is able to generate complex explanations of a certain phenomenon, contrasting variable orientated statistical methods [9]. Therefore, the sensitivity to complex causal relations is another advantage of QCA method contrasting statistical and single caseoriented methods.

Moreover, through this method both numeric and nonnumeric data can be processed, as this method commonly allows data transformation. Relationships between explanatory variables and outcomes can be derived on the basis of numeric, nonnumeric data or their combination. Concerning qualitative data, coding of variables can become an obstacle, as some researchers can tend to use simplified threshold values for them. As a consequence, results can be skewed and not revealing the right causal conditions impacting the outcome.

As the QCA has a different goal and orientation, sometimes its results can be generalized with limitation or cannot be generalized at all. This can be seen as a drawback of this, which is shared with the majority of qualitative research methods. The limited ability to generalize is usually depending on the way how the analysed cases are selected and on the extent to which they represent wider sample.

Besides problems associated with variable coding, QCA is criticized by experts because of instability of causal predictions based on its results [10]. Adding a single case containing extremely different causal conditions than the rest of sample can significantly change the results of QCA, as a consequence of changes in sufficient conditions. However, Ragin himself highlights than QCA application is not sufficient and that it should be accompanied by conscientiously interpreted results [3] on the basis of deep knowledge of the researched problematics. In addition, he makes suggestion to supplement QCA method with other qualitative procedures to come up with robust causal findings.

\section{Basic concepts of QCA}

Originally, the first version of QCA was based on crisp-set relations, so it was called crispset QCA (csQCA). Generally, each case can be attributed two values in csQCA. The values can be either 1 or 0,1 defining membership in the set and 0 referring to non-membership [11]. This means that a certain condition or their combination is either able to result in a certain outcome or is not. Although this technique simplifies complex configurations using principles of Boolean algebra, the reality is more complex. Conditions and outcomes in real world are not always dichotomous, a large variety of sets between full membership and full 
non-membership exists. In fact, results of csQCA could be practically applied with significant limitations. Forthis reason, Ragin deepened his theory to so called fuzzy-set qualitative comparative analysis (fsQCA).

FsQCA enables researchers to classify cases, in which conditions vary to some extent. The decimal values on a scale between 0 and 1 can be used to reflect various degrees of inclusion in a set. In this concept, the values of 0 and 1 can be called as extreme ones reflecting situations of "fully out", or "fully in", a set [12]. Value of 0.5 is usually used as the cross-over point, a threshold [1,7], establishing either membership or non-membership in a set. Fuzzy sets can be continuous ( $0,0.2,0.3$ and so on) or defined by anchor points (e. g. $0,0.33,0.66,1$ in case of anchor fuzzy set with four thresholds). The choice of fuzzy sets depends on theory and it should correspond to differences in case. So if any difference can be distinguished between membership of 0.6 and 0.7 , continuous scoring is not the best option and an anchor fuzzy set with four threshold points can be used to characterize the data. Generally, using the continuous values helps to maintain data more detailed, on the other hand, their interpretation is more complicated contrasting csQCA.

Some new QCA forms are appearing to tackle the main cons of fsQCA, however, they are not so popular yet. One of examples can be multi-value QCA (mvQCA) through which multilevel conditions can be analysed and consequently additional aspect of causal complexity can be captured. Recently, QCA techniques covering also time component have emerged. However, fsQCA remains the most commonly used concept of this method. This fact can be derived even from the number of published papers using QCA [13]. Therefore, the next section of the paper is devoted directly to performing of fsQCA.

\section{Managing fsQCA analysis}

First of all, a dependent variable and independent variables, explaining the dependent one, must be set. In this context, it is important to point out that as QCA deals with middle number of cases, the number of identified explaining variables cannot be too vast. There should be from four to six explaining variables depending on number of selected cases [14]. Consequently, data concerning a field of researcher's interest is collected, then the data matrix is created. Such a matrix represents the main input for fsQCA application.

The raw data need to be calibrated to create a main input for fsQCA application. It means they should be given specific values ranging from 0 to 1 based on theoretical and empirical knowledge. The calibration process of qualitative data is not frequently discussed in literature and in some papers, it lacks transparency. The big challenge for researchers is to calibrate semantic concepts, whose straightforward calibration is not possible. Such a type of information should be calibrated systematically and in a transparent way. However, there are not yet any guidelines for this procedure.

The most common option of dealing with this issue is defining a calibration threshold and decision rules $[1,7]$, which define values being still in a set or yet out of the set. As a result of calibration, data is scaled into set memberships, where values closer to 1 mean "rather in a set", on the other hand, values closer to 0 represent "rather out of a set".

Subsequently, the necessity of a single condition for the outcome must be tested. If a necessary condition exists, it means it is necessary for the presence or absence of outcome, such a condition is excluded from the following analysis. To find out an existence of such a condition, the parameter of consistency needs to be defined [15]. It serves for the same reasons as p-value in regression analysis, so it says to which extent the cases share a certain condition in the outcome. Low consistency of a configuration means that it is not supported by empirical evidence. According to Ragin [1], the threshold value of consistency should be set at 0.9 to assure an acceptable robustness of results. In compliance with qualitative research, the causes of an outcome are understood as combinations of necessary and 
sufficient conditions, that is why, the sufficiency of single condition or their combination for the outcome is analysed as well [16].

All the possible configurations of conditions are transformed into a truth table, in which columns are represented by individual variables, rows stand for individual configurations [17]. Maximum 2k (k stands for number of conditions) can be present in a truth table [18]. Truth tables can be also displayed by Venn diagrams showing all the possible relations between individual conditions. According to the set consistency threshold, the scores are coded, scores below the consistency threshold are coded by 0 illustrating their inconsistency, scores above this value are coded by 1 . Moreover, a frequency threshold should be stated [15] for configurations to be included in the analysis. Frequency threshold refers to number of cases for which a configuration is valid [1, 19]. As fsQCA usually deals with middle- $\mathrm{N}$, this parameter is typically set at one case, if more cases are analysed it is regulated to the value of two.

The configurations in truth tables are further minimized on the basis of Boolean logic. If two configurations lead to the identical outcome and they differ only in one condition, such a condition can be viewed as irrelevant and is excluded [18]. This process is repeated till all the irrelevant conditions are removed away. Afterwards, the highest possible reduction of conditions is reached and makes the results easier to interpret [19]. Lastly, a post-QCA analysis is carried out to interpret the results in the scope of empirical and theoretical knowledge. All the research is evaluated including the practical meaning of found combinations.

\section{Conclusion}

The paper aims at QCA as one of configurative comparative analysis methods. The basic methodological foundations of QCA are provided to give an overview on this causal complexity approach. It follows that the QCA method is rooted in principles of causal asymmetry, conjunctural causation and equifinality. Thanks to them, the analysed cases are considered in a holistic way with the aim to find out which conditions, or their combinations, resulting in a required outcome.

These relations between conditions are evaluated on the basis of logic of necessary and sufficient conditions. Firstly, necessity conditions are tested and alternatively excluded, afterwards, testing of necessity conditions follow. Then all the possible configurations of conditions are displayed in a truth table. Such configurations need to minimalized using Boolean logic to get the highest reduction of conditions allowing easier interpretation of results.

In fact, all the concepts of QCA, including crisp-set and fuzzy-set QCA, bridges quantitative and qualitative research. The QCA method deals with middle sample size, both numerical and nonnumerical data can be used as inputs for this method. Compared to typical qualitative and quantitative methods, QCA generates complex explanations of a certain phenomenon. Obviously, using of QCA has also some limitations, especially in the lack of possible generalization or instability of causal predictions based on QCA.

\section{References}

1. Marx, A., Rihoux, B., \& Ragin, C. (2014). The origins, development, and application of Qualitative Comparative Analysis: the first 25 years. European Political Science Review: EPSR, 6(1), 115. 
2. Rihoux, B et al. (2013). From niche to mainstream method? A comprehensive mapping of QCA application in journal articles from 1984 to 2011. Politicaly Research Quartely, 55(1), 175-184.

3. Ragin, C., \& Zaret, D. (1983). Theory and method in comparative research: Two strategies. Social forces, 61(3), 731-754.

4. Simister, N., Scholz, V. (2017). Qualitative Comparative Analysis (QCA). Intrac. https://www.intrac.org/wpcms/wp-content/uploads/2017/01/Qualitative-comparativeanalysis.pdf

5. Misangyi, V. F., Greckhamer, T., Furnari, S., Fiss, P. C., Crilly, D., \& Aguilera, R. (2017). Embracing causal complexity: The emergence of a neo-configurational perspective. Journal of Management, 43(1), 255-282.

6. Schneider, C. Q., \& Wagemann, C. (2010). Qualitative comparative analysis (QCA) and fuzzy-sets: Agenda for a research approach and a data analysis technique. Comparative Sociology, 9(3), 376-396.

7. Schneider, C. Q., \& Wagemann, C. (2010). Standards of good practice in qualitative comparative analysis (QCA) and fuzzy-sets. Comparative Sociology, 9(3), 397-418.

8. Kouba, K. (2012). Kvalitativní srovnávaci analýza (QCA) a konfigurativní metody v politologii. Úvod do studia politiky. Slon, 468-507.

9. Kahwati, L., Jacobs, S., Kane, H., Lewis, M., Viswanathan, M., \& Golin, C. E. (2016). Using qualitative comparative analysis in a systematic review of a complex intervention. Systematic reviews, 5(1), 82.

10. George, A., Bennett, A. (2004). Case studies and theory development in the social sciences. Studies in Comparative International Development, 41(2), 391-408

11. Skaaning, S. E. (2011). Assessing the robustness of crisp-set and fuzzy-set QCA results. Sociological Methods \& Research, 40(2), 391-408.

12. Vassinen, A. (2012). Configurational explanation of marketing outcomes. Aalto University Schoold of Economics.

13. Roig-Tierno, N., Gonzalez-Cruz, T. F., \& Llopis-Martinez, J. (2017). An overview of qualitative comparative analysis: A bibliometric analysis. Journal of Innovation \& Knowledge, 2(1), 15-23.

14. Berg-Schlosser, D. (2008). Comparative research design: case and variable selection. Configurational Comparative Methods. SAGE Publications

15. Woodside, A. (2013). Moving beyond multiple regression analysis to algorithms for adoption of a paradigm shift from symmetric to asymmetric thinking in data analysis and crafting theory. Journal of Business Research, 66(4), 463-472

16. Munck, G. L. (2004). Tools for qualitative research. Rethinking social inquiry. Diverse tools, shared standards. Rowman and Littlefield Publishers.

17. Fiss, P. C. (2011). Building better causal theories: A fuzzy set approach to typologies in organization research. Academy of management journal, 54(2), 393-420..

18. Ragin, C. C. (2009). Reflections on casing and case-oriented research. The Sage handbook of case-based methods. 522-534.

19. Woodside, A. G., \& Zhang, M. (2012). Identifying x-consumers using causal recipes:"Whales" and "jumbo shrimps" casino gamblers. Journal of Gambling Studies, 28(1), 13-26. 